\title{
Use of smartphone and perception towards the usefulness and practicality of its medical applications among healthcare workers in Saudi Arabia
}

\author{
Mostafa A. Abolfotouh ${ }^{1 *}$ (D), Ala'a BaniMustafa ${ }^{2}$, Mahmoud Salam² ${ }^{2}$ Mohammed Al-Assiri ${ }^{2}$, Bader Aldebasi ${ }^{1}$ and \\ Ibraheem Bushnak ${ }^{1}$
}

\begin{abstract}
Background: In Saudi Arabia, healthcare industry is undergoing major expansions to meet the demand of rapidly growing healthcare needs. The aims of this study were; (1) to assess the pattern of smartphone use in healthcare facilities, and (2) to determine perception towards its use among healthcare workers.

Method: A cross-sectional survey of 351 healthcare workers (HCWs) at King Abdulaziz Medical City (KAMC) in Riyadh, Saudi Arabia was conducted, from October to November 2016, using a previously validated perception domain to measure perception towards usefulness ( 5 statements) and practicality ( 5 statements) of smartphones in clinical settings. Pattern of use of smartphones and health-related applications in healthcare facilities was also investigated. Logestic regression models were applied to identify the predictors of smartphone use and installation of health-related applications for use in healthcare facilities. Significance was considered at $p$-value of $<0.05$.

Results: Utilization rate of smartphone was $42.3 \%$, and only $6.1 \%$ of all healthcare providers reported always using applications in their practice. Reasons for use were: as a source of drug information (69.8\%), for disease diagnosis (56.4\%), to access medical websites (42.5\%), to review guidelines and protocols related to healthcare (34.1\%), for procedure documentation (23.5\%), and as a source of patients education materials (22.3\%). Perceptions of HCWs towards smartphone use was less than satisfactory (Overall percentage mean score $=60.4 \pm 18.7$ ), with only $11.6 \%$ reporting positive perception. After adjusting for possible confounders, the total perception mean score was a significant predictor of both smartphone use $(\beta=0.033, p<0.001)$ and medical applications installation $(\beta=0.033$, $p<0.001$ ). Installation of medical applications was also predicted by being a physician $(\beta=0.008, p=0.024)$.

Conclusion: Smartphone utilization in healthcare facilities by HCWs in Saudi Arabia is low. This could be attributed to their less than satisfactory level of perception towards its use. Smartphone use and installation of medical applications for use in health facilities were predicted by perceived usefulness and practicality of its use. Intervention from higher health authorities is necessary to enforce the importance of smartphone use in clinical practice. Conduction of further studies on the impact of smart phone use on the healthcare quality in Saudi Arabia is recommended.
\end{abstract}

Keywords: Attitude, Healthcare facility, Health-related applications, Pattern of use, Smartphone use

\footnotetext{
*Correspondence: mabolfotouh@gmail.com

${ }^{1}$ Research Training \& Development Section, King Abdullah International

Medical Research Center (KAIMRC), King Saud bin-Abdulaziz University for

Health Sciences (KSAU-HS), King Abdulaziz Medical City, Ministry of National

Guard- Health Affairs, Riyadh 11426, Saudi Arabia

Full list of author information is available at the end of the article
}

(c) The Author(s). 2019 Open Access This article is distributed under the terms of the Creative Commons Attribution 4.0 International License (http://creativecommons.org/licenses/by/4.0/), which permits unrestricted use, distribution, and reproduction in any medium, provided you give appropriate credit to the original author(s) and the source, provide a link to the Creative Commons license, and indicate if changes were made. The Creative Commons Public Domain Dedication waiver (http://creativecommons.org/publicdomain/zero/1.0/) applies to the data made available in this article, unless otherwise stated. 


\section{Background}

Information technology (IT) softwares in healthcare provide clinicians with health-related information and tools, such as clinical decision supportive systems. These softwares are supposed to reduce potential medical errors in any healthcare facility and improve the quality of patient care [1]. The rising demand to improve the healthcare processes via technical products and services has resulted in a huge development of wireless technologies worldwide [2].

Smartphone has started as a multi-functional mobile phone with advanced features. Some applications in smartphones are health-related, and may be useful in a wide variety of clinical aspects. Proper utilization of these applications vary in degrees of success, and this is based on being easy-to-learn and use [3]. A previous study reported that the utilization of smartphones had provided practitioners with immediate access to medical and health information, and this had a positive impact on a healthcare system. This technology has reduced numbers of medical errors and improved decisionmaking, thus improving the telemedicine communication between the hospital staff [4].

Smartphone applications have been widely accepted as training and information tools. The percentage of health professionals using smart phones has risen from 66 to 90\% in 2012 [5]. A digital survey examining smartphone and its application use was conducted by Accreditation Council for Graduate Medical Education (ACGME) in California, showed that more than $85 \%$ of respondents used a smartphone, of which the iPhone was the most popular (56\%). Over half of the respondents reported using applications in their clinical practice; the most frequently used application types were; drug guides (79\%), medical calculators (18\%), coding and billing applications (4\%) and pregnancy wheels (4\%). The most commonly requested application types were textbook/ reference materials (average response: 55\%), classification/ treatment algorithms (46\%) and general medical knowledge (43\%) [6].

Davis in 1989 [7] formulated the Technology Acceptance Model (TAM) as a mechanism to explain and predict consumer's acceptance of information technology and information system (IT/IS) [7]. There are originally five elements of TAM: perceived usefulness (PU), perceived ease of use (PEOU), attitude toward using, behavioral intention (BI) to use, and actual system use $[2,8]$. The TAM model suggests that the decision to adopt a system is based on two main factors: Perceived Usefulness (PU) and Perceived Ease of Use (PEOU). PU is the extent a person feels that utilization of a specific technology would improve his/her job performance, while PEOU refers to the degree a person feels that utilization of a particular technology requires no effort $[2,7]$.
In Saudi Arabia, the healthcare industry is undergoing major expansions to meet the demand of rapidly growing healthcare needs. A user-acceptance study utlizing a TAM based questionnaire was recently conducted in AlQassim focusing on the perception and readiness of health providers towards mobile health applications [9]. However, locally conducted studies didn't fully measure the combined factors affecting perception, attitude and practice using the TAM. On other side, another study was counducted in Almadinah Almunawwarah trying to assess patterns of smart phones usage among female medical students which focused on female population and assessed the side effects of smart phones [10]. At King Abdulaziz Medical City (KAMC), it is estimated that the number of healthcare providers exceeds 7000 between the central, eastern and western regions. However, rules and regulations regarding using smartphones at hospital by healthcare providers in Saudi Arabia have not yet existed. Accordingly, it was expected that the feedback provided by these professionals would provide better undertsnading of the efficiency and pacticality of a number of currently used health applications. Findings would contribute in assessing the needs for upcoming development of health related applications for staff education, thus providing instruction and guidence for optimal use of smart phone's apps to enhance clinical practice. The aims of this study were; (1) to assess the pattern of smartphone use in healthcare facillities [time, frequency and purpose behind using health-related applications in smart phones], (2) to determine perception towards its use and (3) to identify factors influencing the usage of smartphone in clinical practice and installation of medical applications, among healthcare workers in a tertiary care setting.

\section{Methods \\ Study area/setting}

King abdulaziz medical city (KAMC), Ministry of National Guard-Health Affiars (MNG-HA), in Riyadh, is a distinguished healthcare provider, with the bed capacity of 1501 beds in addition to 25 beds allocated for expected surgical operations for admission of emergency cases. Since its inauguration in February 2001; and within a short period, KAMC has passed the requirements for accreditation under the (JCI) Joint Commission International standards with excellent performance in December 2006.

\section{Study subjects}

All healthcare workers (HCWs) working in departments of medicine, nursing, laboratories, respiratory therapy, radiology, nutrition, paramedics, clinical pharmacists, auxiliiaries, sterilizing unit technicians, infection control practitioners. 


\section{Study design}

Cross sectional survey.

\section{Sample size and sampling technique}

The Accreditation Council for Graduate Medical Education (ACGME) in California found that $56 \%$ of medical providers use smart phone applications in their clinical practice [6]. Assuming a $50 \%$ favourable perception, a margin of error of 5\%, and $95 \%$ confidence level, the estimated sample size that would statistically be convenient in this study was 378 participants. To compensate for an estimated $25 \%$ drop out rate due to incomplete surveys and withdrawals, 470 surveys were distributed. Those who responded were 351 HCWs, with a response rate of $75 \%$.The nature of the project design entitled a convenient sampling technique. This non-probabilty sampling approach voluntarily involved HCWs who were available to make the sample a better representative of the entire population of KAMC in Riyadh ( $4000 \mathrm{HCWs})$. Careful attention was made to ensure various disciplines (medicine, nursing, etc.) participate in comparable sample sizes.

\section{Data collection}

The survey was composed of three main sections:

1) Demographic characteristics of the study sample: A number of sample characteristics were collected as independent variables or exposures. These include; gender (Male / female), age (in years), nationality (Saudi/Non Saudi), education (BSN/ MSN/PHD /Tech /Diploma), occupation (Employee / Student), job title (Physician, Nurse, etc.), discipline (Medicine, Nursing etc.), and experience (in years),

2) Pattern of usage and purpose domain $[4,6,11]$ : Possesion of smart phones, health-related applications or any other portable devices, frequency of usage, time spent in hours daily, etc..

3) Perceived Usefulness and Perceived Ease of Use. A previously validated domain that measures the perception towards the usefulness (5 statements) and practicality ( 5 statements) of smart phones in clinical settings was used [7, 9]. Perception score was calculated for the responses to the 10 statements by using 4-point Likert type scale ranging from 0 to 3 points; 3 points for strongly agree, on positive attitude sentence, to 0 point for "strongly disagree". The total score for each $\mathrm{HCW}$ was calculated by summing scores for all responses, and then a percentage score was calculated. This percentage score was categorized into positive $(>75 \%)$, neutral $(50-75 \%)$ and negative $(<50 \%)$ perception.
The investigators distributed an annonymous self administered English based survey inside an envelope with a cover letter. Each envelope was handed to the HCW at his/her department. HCWs are Saudis and expatriates of different nationalities, with Arab and non-Arab speakers, yet English language is the official language of communication among the HCWs at KAMC. Study particpants were expected to fill the survey and return it in the envelope sealed with no identifiers. The cover letter served as the front page that explained the purpose of the study and invited the HCW to particpate voluntarly and at his/her own leasure. The envelope was not recognized by the hospital or investigator. The cover letter assured the particpant that his/her feedback wont affect their work evaluation, work status, and salary. The collection of data was framed with confidentiality in a matter where the participant's name and/or contact information were not identified or traced by anyone. No written consent was sought as approved by the IRB. The study was approved by the IRB of the Ministry of National Guard-Health Affairs (Ref. \# RC16/002/R). This study was conducted in accordance with the Declaration of Helsinki. To test the feasibility of the study and the reliability and validity of data collection tool, a pilot study was performed on 20 randomly selected participants who were subsequently excluded from the main study.

\section{Data analysis}

SPSS software Ver. 24 was used for data entery and analysis. Descriptive statistics such as; mean, standard deviation, frequency and percentages of all independent variables were used. Analytic statistics were uded to test associations of the HCWs' perception towards smartphone use with both smartphone use and instalation of medical applications. Chi-square test was used for qualitative data, while student t-test and ANOVA were applied for quantitative data. To predict the significant predictors of HCWs' smartphone use and installation of medical applications, logestic regression analyses were applied, with age group, nationality, education level, marital status, job title, working experience in years and total perception mean score as the independent variables. Significance was considered at $p$-value $<0.05$.

\section{Results}

A total of 351 healthcare workers responded to a survey to assess their perception towards the use of smartphone in clinical practice, with a response rate of $93 \%$. The majority of those were non-Saudis (90.3\%), married (55.2\%) female $(88.3 \%)$, nurses $(76.6 \%)$, aged 30 to 40 years (40.5\%), and working in medical departments (49.7\%), Table 1. 


\section{Ownership and use of smartphone}

Almost all healthcare workers owned one or more smartphones (96.6\%), but only $42.3 \%$ utilize it in healthcare practice. Less than two-thirds of all healthcare providers $(60.1 \%)$ reported using other appliances such as; IPAD (43.6\%), tablet (7.5\%) and others (10.3\%), and less than one-half installed applications to use in clinical practice (45.5\%). Regarding the frequency of use of smartphone, only $6.1 \%$ of all healthcare providers

Table 1 Demographic characteristics of healthcare workers

\begin{tabular}{|c|c|c|}
\hline Characteristics & No $(n=351)$ & $\%$ \\
\hline \multicolumn{3}{|l|}{ Gender } \\
\hline Male & 41 & 11.7 \\
\hline Female & 310 & 88.3 \\
\hline \multicolumn{3}{|l|}{ Age group (yrs) } \\
\hline $20-29$ & 86 & 25.6 \\
\hline $30-39$ & 136 & 40.5 \\
\hline $40+$ & 114 & 33.9 \\
\hline Mean (SD) & $36.1 \pm 8.7$ & \\
\hline \multicolumn{3}{|l|}{ Education } \\
\hline Diploma & 89 & 25.7 \\
\hline BSN & 247 & 71.4 \\
\hline MSN/PHD & 10 & 2.9 \\
\hline \multicolumn{3}{|l|}{ Nationality } \\
\hline Saudi & 34 & 9.7 \\
\hline Non-Saudi & 317 & 90.3 \\
\hline \multicolumn{3}{|l|}{ Marital status } \\
\hline Single & 145 & 41.7 \\
\hline Married & 192 & 55.2 \\
\hline Divorced/ Widowed & 11 & 3.1 \\
\hline \multicolumn{3}{|l|}{ Job title } \\
\hline Physician & 8 & 2.3 \\
\hline Nurse & 268 & 76.6 \\
\hline Pharmacist & 2 & 0.6 \\
\hline Lab. technician & 29 & 8.3 \\
\hline Others & 43 & 12.2 \\
\hline \multicolumn{3}{|l|}{ Workplace } \\
\hline Medical & 172 & 49.7 \\
\hline Surgical & 21 & 6.1 \\
\hline Lab. & 34 & 9.8 \\
\hline Pharmacy & 1 & 0.3 \\
\hline Others & 118 & 34.1 \\
\hline \multicolumn{3}{|c|}{ Work experience (in years) } \\
\hline$<10$ & 160 & 47.2 \\
\hline $10-19$ & 127 & 37.5 \\
\hline 20 or more & 52 & 15.3 \\
\hline Mean (SD) & $11.6 \pm 7.3$ & \\
\hline
\end{tabular}

reported always using the application in their practice, and $26.2 \%$ of them using it sometimes. The reasons for use of smartphone in healthcare practice were: as a source of drug information (69.8\%), for disease diagnosis (56.4\%), to access medical websites (42.5\%), to review guidelines and protocols related to healthcare (34.1\%), for procedure documentation (23.5\%), and as a source of patients education materials (22.3\%), Table 2.

\section{Perception towards importance of smartphone use}

Table 3 shows that beliefs of healthcare providers in smartphone use in terms of usefulness and practicality was less than satisfactory (Overall percentage mean score $=60.4 \pm$ 18.7), only $11.6 \%$ reporting positive attitude. Percentage mean scores of $58.6 \pm 19.7$ and $62.5 \pm 19.9$ were shown for

Table 2 Ownership and utilization of smartphones by healthcare workers

\begin{tabular}{|c|c|c|}
\hline & No. & $\%$ \\
\hline \multicolumn{3}{|l|}{ Own smartphone? ${ }^{\mathrm{a}}$} \\
\hline None & 12 & 3.4 \\
\hline Iphone & 169 & 48.4 \\
\hline Android & 168 & 48.2 \\
\hline Nokia & 4 & 1.2 \\
\hline Blackberry & 2 & 0.6 \\
\hline Others & 5 & 1.4 \\
\hline Utilization of smartphone in healthcare & 146 & 42.3 \\
\hline \multicolumn{3}{|l|}{ Use of other appliences ${ }^{a}$} \\
\hline No & 127 & 39.9 \\
\hline Tablet & 24 & 7.5 \\
\hline IPAD & 139 & 43.6 \\
\hline Others & 33 & 10.3 \\
\hline Installed medical applications? & 157 & 45.5 \\
\hline Frequency of use & $(N=309)$ & $\%$ \\
\hline Never & 142 & 46.0 \\
\hline Rarely & 67 & 21.7 \\
\hline Sometimes & 81 & 26.2 \\
\hline Always & 19 & 6.1 \\
\hline Reasons for use $^{a}$ & No $(n=179)$ & $\%$ \\
\hline Drug information & 125 & 69.8 \\
\hline $\begin{array}{l}\text { Clinical score systems (coma scale, } \\
\text { APACHE, pain score ...) }\end{array}$ & 33 & 18.4 \\
\hline Disease diagnosis & 101 & 56.4 \\
\hline Procedure documentation & 42 & 23.5 \\
\hline Access medical web sites & 76 & 42.5 \\
\hline $\begin{array}{l}\text { Review guideline and protocol } \\
\text { related to healthcare }\end{array}$ & 61 & 34.1 \\
\hline Patients' education materials & 40 & 22.3 \\
\hline Others & 12 & 6.7 \\
\hline
\end{tabular}

a--data are not mutually exclusive 
Table 3 Perception of healthcare workers towards usefulness and practicality of use of smart phone in clinical settings

\begin{tabular}{|c|c|c|c|c|c|}
\hline & Perception towards smartphone use in healthcare & $\begin{array}{l}\text { Strongly } \\
\text { disagree }\end{array}$ & Disagree & Agree & $\begin{array}{l}\text { Strongly } \\
\text { agree }\end{array}$ \\
\hline & & No.(\%) & No.(\%) & No.(\%) & No.(\%) \\
\hline & (A) Usefulness & & & & \\
\hline 1 & Medical apps on smart phone improve my tracking of patient condition and performance & 20(6.2) & $67(20.6)$ & 212(65.2) & 26(8.0) \\
\hline 2 & Medical apps on smart phone save the time and efforts of healthcare provider & $18(5.5)$ & $60(18.4)$ & 213(65.3) & $35(10.8)$ \\
\hline 3 & Medical apps on smart phone rapidly retrieve of the information from the patient. & 19(5.9) & $79(24.7)$ & 197(61.6) & 25(7.8) \\
\hline 4 & $\begin{array}{l}\text { Medical apps on smart phone allow healthcare provider to follow up the patient condition } \\
\text { from outside of the hospital }\end{array}$ & $25(7.7)$ & $86(26.6)$ & 181(56.0) & $31(9.7)$ \\
\hline 5 & $\begin{array}{l}\text { Medical apps on smart phone enable healthcare provider to get the information of the patient } \\
\text { quickly (through the software) }\end{array}$ & 19(5.9) & $51(15.8)$ & 216(67.1) & $36(11.2)$ \\
\hline & Percentage mean score & $58.6 \pm 19.7$ & & & \\
\hline & (B) Practicality & & & & \\
\hline 6 & Learning to operate and use medical apps on smart phone would be easy for me & 15(4.6) & 29(9.0) & 234(72.2) & $46(14.2)$ \\
\hline 7 & I would find it easy get medical apps on smart phone to do what I want it to do & 16(5.0) & $55(17.1)$ & 218(67.7) & $33(10.2)$ \\
\hline 8 & My interaction with medical apps on smart phone would be clear and understandable & 16(5.0) & $45(13.9)$ & 232(71.8) & 30(9.3) \\
\hline 9 & I would find medical apps on smart phone to be flexible to interact with & 16(4.9) & 39(12.0) & 236(72.8) & $33(10.3)$ \\
\hline 10 & It would be easy for me to become skillful at using medical apps on smart phone & 19(5.8) & $44(13.5)$ & 224(68.9) & 38(11.8) \\
\hline & Percentage mean score & $62.5 \pm 19.9$ & & & \\
\hline & Overall percentage mean score & $60.4 \pm 18.7$ & & & \\
\hline & & Negative & Neutral & Positive & \\
\hline & Levels of perception toward smartphone use & No.(\%) & No.(\%) & No.(\%) & \\
\hline & Usefulness & $82(26.0)$ & $201(63.8)$ & $32(10.2)$ & \\
\hline & Practicality & $54(16.9)$ & $226(70.8)$ & $39(12.3)$ & \\
\hline & Overall perception of smartphone use & $50(16.0)$ & $226(72.4)$ & 36(11.6) & \\
\hline
\end{tabular}

usefulness and practicality respectively, with only 10.2 and $12.3 \%$ reporting positive attitude towards the importance of smartphone use.

\section{Association between perception towards smartphone use and its use}

Table 4 shows the association between the level of perception to smart phone use and prevalence of its use and installation. It shows that the prevalence of smartphone use was $26 \%$ among subjects with negative perception towards its use. This figure was doubled $(43.2 \%, \mathrm{OR}=2.17, p=0.025)$ and tripled $(74.3 \%$, $\mathrm{OR}=8.22, p<0.001)$, among those with neutral and positive perceptions respectively. This finding was evident for both perception domains; usefulness and practicality. Likewise, the prevalence of installation of medical applications was $28 \%$ among HCWs with negative perception towards smartphone use, and this figure was doubled and tripled as we shifed from those with negative perception (20.0\%) to neutral (48.4\%, OR $=3.76, \quad p<0.001)$ and positive $(66.7 \%$, $\mathrm{OR}=8.0, p<0.001)$ perceptions. This finding was evident for both perception domains. After adjusting for possible confounders, the total perception mean score was a significant predictor of both smartphone use $(\beta=0.033, p<0.001)$ and installation of medical applications $(\beta=0.033, p<0.001)$. Installation of medical applications was also predicted by physicians $(\beta=$ $0.771, p=0.024)$, Table 5 .

\section{Discussion}

Increasing use of smartphone applications among healthcare professionals has gained wide acceptance as a training and information tool $[12,13]$. It had been estimated that the percentage of medical professionals using smartphones would reach 66-90\% in 2012 [14]. In our study, almost all health providers owned one or more smartphones (96.6\%), but only $42.3 \%$ utilized it in healthcare practice. A previous survey examining smartphone and its application use was conducted by Accreditation Council for Graduate Medical Education (ACGME) in California [6] showed that more than 85\% of respondents used a smartphone, of which the iPhone was the most popular (56\%). In the present study, $60.1 \%$ reported using other appliances such as; IPAD (43.6\%), tablet $(7.5 \%)$ and others (10.3\%). 
Table 4 Rate of smartphone use (\%) and installation of medical applications (\%) according to levels of perception towards use

\begin{tabular}{|c|c|c|c|c|c|c|c|c|}
\hline & \multicolumn{4}{|c|}{ Use of smartphone } & \multicolumn{4}{|c|}{ Installation of medical application(s) } \\
\hline & No. & $\%$ & OR $[95 \% \mathrm{Cl}]$ & $p$-value & No. & $\%$ & OR & $p$-value \\
\hline \multicolumn{9}{|l|}{ Usefulness } \\
\hline Negative & 21 & 25.6 & 1 & & 23 & 28.0 & 1 & \\
\hline Neutral & 93 & 47.2 & $2.59[1.47-4.59]$ & 0.001 & 103 & 52.0 & $2.78[1.59-4.85]$ & $<0.001$ \\
\hline \multirow[t]{2}{*}{ Positive } & 23 & 74.2 & $8.35[3.25-21.49]$ & $<0.001$ & 18 & 56.3 & $3.30[1.41-7.71]$ & 0.005 \\
\hline & \multicolumn{4}{|c|}{$x Z_{L T}=23.26, p<0.001$} & \multicolumn{4}{|c|}{$X 2_{L T}=12.37, p<0.001$} \\
\hline \multicolumn{9}{|l|}{ Practicality } \\
\hline Negative & 14 & 25.9 & 1 & & 9 & 16.7 & 1 & \\
\hline Neutral & 99 & 44.6 & 2.30[1. 18-4.47] & 0.012 & 112 & 50.5 & $5.09[2.38-10.91]$ & $<0.001$ \\
\hline \multirow[t]{2}{*}{ Positive } & 26 & 68.4 & $6.19[2.48-15.47]$ & $<0.001$ & 25 & 64.1 & 8.93[3.39-23.55] & $<0.001$ \\
\hline & \multicolumn{4}{|c|}{$X 2_{\mathrm{LT}}=16.14, p<0.001$} & \multicolumn{4}{|c|}{$X 2_{L T}=22.88, p<0.001$} \\
\hline \multicolumn{9}{|l|}{ Overall } \\
\hline Negative & 13 & 26.0 & 1 & & 10 & 20.0 & 1 & \\
\hline Neutral & 96 & 43.2 & $2.17[1.09-4.30]$ & 0.025 & 108 & 48.4 & $3.76[1.79-7.88]$ & $<0.001$ \\
\hline \multirow[t]{2}{*}{ Positive } & 26 & 74.3 & $8.22[3.07-22.06]$ & $<0.001$ & 24 & 66.7 & $8.00[3.00-21.32]$ & $<0.001$ \\
\hline & \multicolumn{4}{|c|}{$X 2_{L T}=18.44, p<0.001$} & \multicolumn{4}{|c|}{$X 2_{\mathrm{LT}}=19.63, p<0.001$} \\
\hline
\end{tabular}

$\mathrm{X}^{2}$--- Chi square for linear trend, OR---Odds ratio

The most frequently requested application types were textbook/ reference materials (average response $=$ $55 \%)$, classification/ treatment algorithms (46\%) and general medical knowledge (43\%) [6]. In our study, nearly half of all healthcare providers installed applications to use in clinical practice (45.5\%). Only $6.1 \%$ of all healthcare providers always use the application in their practice, and $26.2 \%$ of them use it sometimes. The reasons for use of smartphone in healthcare practice were: as a source of drug information (69.8\%), for disease diagnosis (56.4\%), to access medical websites $(42.5 \%)$, to review guideline and protocol related to healthcare (34.1\%), for procedure documentation $(23.5 \%)$, and as a source of patients education materials $(22.3 \%)$.

\section{Perception of HCWs towards usefullness and practicality} of use of smartphones

Technology Acceptance Model (TAM) was formulated to assess consumer's level of acceptance of information technology and information system (IT/IS) [2, 7]. In the present study, the results showed that beliefs of healthcare providers in smartphone use - in terms of usefulness and practicality - was less than satisfactory, with only $11 \%$ reporting positive attitude. This finding might be attributed to the non-existence of a formal system implemented in the healthcare facilities in Saudi Arabia that allows healthcare providers be convinced of use of smarphone as a new technology that would impact the healthcare quality, by providing practitioners with immediate access to medical and health information.

Table 5 Predictors of use of smartphones and installation of medical applications among HCWs in Saudi Arabia

\begin{tabular}{|c|c|c|c|c|c|c|}
\hline \multirow[t]{2}{*}{ Independent variables } & \multicolumn{3}{|c|}{ Use of smartphone } & \multicolumn{3}{|c|}{ Install med. Applications } \\
\hline & $\beta$ & SE & $p$-value & $\beta$ & SE & $p$-value \\
\hline Gender & .278 & .39 & .474 & .600 & .390 & .124 \\
\hline Age Group & .028 & .266 & .916 & .281 & .264 & .287 \\
\hline Nationality & .357 & .48 & .46 & -.062 & .471 & .895 \\
\hline Diploma vs others & -.333 & .314 & .288 & -.354 & .311 & .255 \\
\hline Single vs others & -.179 & .278 & .518 & .208 & .274 & .449 \\
\hline physicians vs others & -.432 & .331 & .192 & .771 & .342 & $.024^{* *}$ \\
\hline Working experience (yrs) & -.040 & .029 & .172 & -.045 & .028 & .113 \\
\hline Total Perception mean score [\%] & .033 & .009 & $<.001^{* *}$ & .033 & .008 & $<.001^{* *}$ \\
\hline Constant & -1.457 & .750 & .052 & -2.955 & .760 & .000 \\
\hline
\end{tabular}

**---Statistically significant 
This technology has resulted in reduced numbers of medical errors, improved decision-making, and upgrading the telemedicine communication among hospital staff [4]. In the present study, use of smartphone in healthcare was reported by less than one-half of all healthcare workers. The use of smartphone in healthcare was predicted by only the level of perception of HCWs towards the practicality and usefulness of its use. Installation of medical applications by HCWs was positively influenced by both the favourable perception as well as the physicians. Nurses' use of smartphones for work purposes was positively associated with their intention to use smartphones for work purposes, perceived work productivity and perceived quality of care [15]. Prior research has also shown an association between attitude toward HITs and intention to use HITs [16-18]. This finding supports IT consumerization theory and provides empirical support for the argument that nurses' use of smartphones can improve work productivity [19-21] and enhance the quality of care rendered to patients [19, 22].

This study has some limitations. One of these limitations is that the causal association betweeen the use of smartphone and/or installation of medical applications and other variables could not be guaranteed because of the cross sectional design of the study. Second limitation is that the results could not be generalized being the results of a single health facility.

\section{Conclusions}

In conclusion, smartphone use is not a common practice among HCWs in Saudi Arabia. This could be attiributed to their less than satisfactory level of perception towards the use of smart phones in terms of its usefulness and practicality. There must be a necessary intervention from higher health authorities to enforce the importance of use of smartphones in clinical practice. Conduction of further studies on the impact of smart phone use on the healthcare quality in Saudi Arabia is recommended. Such use can improve HCWs' perceived work productivity and perceived quality of care rendered to patients.

\section{Abbreviations \\ ACGME : The Accreditation Council for Graduate Medical Education; Apps: Applications; HCWs: Healthcare workers; HIT: Health information technology; IRB: Institutional Review Board; IT: Information technology; IT/ IS: Information technology and information system; KAMC: King Abdulaziz Medical city; MNG-HA: Ministry of National Guard-Health Affairs; PEOU: Perceived Ease of Use; PMS: Percentage mean score; PU: Perceived Usefulness; TAM: Technology acceptance model}

\section{Acknowledgments}

This study was initiated and supported by King Abdullah International Medical Research Center (KAIMRC), King Saud bin-Abdulaziz University for Health Sciences, Ministry of National Guard -Health Affairs, KSA. The final draft of the manuscript was English language edited by Macmillan Science Communication.

\section{Authors' contributions}

MAA \& ABM contributed to concept development, manuscript preparation and final writing, MA and MS contributed to concept development and data collection, and BA and EB Contributed to research proposal writing, data collection, analysis and interpretation, and manuscript drafting. All authors read and approved the final manuscript.

\section{Funding}

None

\section{Availability of data and materials}

Most of the data supporting our findings is contained within the manuscript and all others, excluding identifying/confidential respondent data should, will be shared upon request. The corresponding author should be contacted to access the data on reasonable request.

\section{Ethics approval and consent to participate}

This study was approved by the institutional review board of the MNG-HA in Riyadh, Saudi Arabia (Ref. \# RC16/002/R). Participation in this study was voluntary. HCWs were assured that their feedback would not affect their performance evaluations, work status or salaries. No written consent was sought, as there were no personal identifiers on the questionnaires, and this was approved by the IRB. Submission of responses to the questionnaire was considered to constitute implied consent. The voluntary nature of participating in the survey was made explicit and unambiguous in the cover letter. The investigators did not coerce or entice anyone to complete the questionnaire. Any participant could decline to return the questionnaire.

\section{Consent for publication \\ Not applicable.}

\section{Competing interests}

The corresponding author (Mostafa Abolfotouh) is an Associate Editor for BMC Health Service Research, and he had no role in the editorial process for this manuscript. All authors declare that they have no competing interests.

\section{Author details}

${ }^{1}$ Research Training \& Development Section, King Abdullah International Medical Research Center (KAIMRC), King Saud bin-Abdulaziz University for Health Sciences (KSAU-HS), King Abdulaziz Medical City, Ministry of National Guard- Health Affairs, Riyadh 11426, Saudi Arabia. ${ }^{2}$ Research Office, King Abdullah International Medical Research Center (KAIMRC), King Saud bin-Abdulaziz University for Health Sciences (KSAU-HS), King Abdulaziz Medical City, Ministry of National Guard- Health Affairs, Riyadh 11426, Saudi Arabia.

Received: 1 April 2019 Accepted: 10 September 2019

Published online: 12 November 2019

\section{References}

1. Putzer GJ, Park Y. The effects of innovation factors on smartphone adoption among nurses in community hospitals. Perspect Health Inf Manag. 2010;7:1b (Winter).

2. Wan Ismail WK, Kit H, Chan P, Buhari N, Muzaini A. Acceptance of smartphone in enhancing patient-caregivers relationship. J Technol Manag Innov. 2012;7(3):71-9.

3. Pandey A, Hasan S, Dubey D, Sarangi S. Smartphone apps as a source of cancer information: changing trends in health information-seeking behavior. J Cancer Educ. 2013;28(1):138-42.

4. Payne KF, Wharrad H, Watts K. Smartphone and medical related app use among medical students and junior doctors in the United Kingdom (UK): a regional survey. BMC Med Inf Decis Mak. 2012;12(1):121.

5. Dubey D, Amritphale A, Sawhney A, Amritphale N, Dubey P, Pandey A. Smart phone applications as a source of information on stroke. J Stroke. 2014;16(2):86.

6. Franko Ol, Tirrell TF. Smartphone app use among medical providers in ACGME training programs. J Med Syst. 2012;36(5):3135-9.

7. Davis FD. Perceived usefulness, perceived ease of use, and user acceptance of information technology. MIS Q. 1989;13:319-40.

8. Wu JH, Wang SC. What drives mobile commerce?: an empirical evaluation of the revised technology acceptance model. Inf Manag. 2005;42(5):719-29. 
9. Abu-Dalbouh HM. A questionnaire approach based on the technology acceptance model for mobile tracking on patient progress applications. J Comput Sci. 2013;9(6):763-70.

10. Jamal A, Sedie R, Haleem KA, Hafiz N. Patterns of use of 'smart phones' among female medical students and self-reported effects. J Taibah Univ Med Sci. 2012;7(1):45-9.

11. Koehler N, Vujovic O, McMenamin C. Healthcare professionals' use of mobile phones and the internet in clinical practice. J Mob Technol Med. 2013;2(1):3-13.

12. Oehler RL, Smith K, Toney JF. Infectious diseases resources for the iPhone. Clin Infect Dis. 2010;50(9):1268-74.

13. Hawkes CP, Walsh BH, Ryan CA, Dempsey EM. Smartphone technology enhances newborn intubation knowledge and performance amongst paediatric trainees. Resuscitation. 2013;84(2):223-6.

14. Senior K. Smart phones: new clinical tools in oncology? Lancet Oncol. 2011;12(5):429-30

15. Bautista JR, Rosenthal $S$, Lin $\Pi$, Theng YL. Predictors and outcomes of nurses' use of smartphones for work purposes. Comput Hum Behav. 2018:84:360-74.

16. Park Y, Chen JV. Acceptance and adoption of the innovative use of smartphone. Ind Manag Data Syst. 2007;107(9):1349-65.

17. Putzer GJ, Park Y. Are physicians likely to adopt emerging mobile technologies? Attitudes and innovation factors affecting smartphone use in the southeastern United States. Perspect Health Inform Manag. 2012;9:1b (Spring).

18. Wu L, Li JY, Fu CY. The adoption of mobile healthcare by hospital's professionals: an integrative perspective. Decis Support Syst. 2011:51(3):587-96.

19. Bautista JR, Lin TT. Sociotechnical analysis of nurses' use of personal mobile phones at work. Int J Med Inform. 2016;95:71-80.

20. Mobasheri MH, King D, Johnston M, Gautama S, Purkayastha S, Darzi A. The ownership and clinical use of smartphones by doctors and nurses in the UK: a multicentre survey study. BMJ Innovations. 2015;1(4):174-81.

21. Moore $\mathrm{S}$, Jayewardene $\mathrm{D}$. The use of smartphones in clinical practice. Nurs Manag. 2014;21(4):18-22. https://doi.org/10.7748/nm.21.4.18.e1225.

22. Chiang KF, Wang HH. Nurses' experiences of using a smart mobile device application to assist home care for patients with chronic disease: a qualitative study. J Clin Nurs. 2016;25(13-14):2008-17.

\section{Publisher's Note}

Springer Nature remains neutral with regard to jurisdictional claims in published maps and institutional affiliations.

Ready to submit your research? Choose BMC and benefit from:

- fast, convenient online submission

- thorough peer review by experienced researchers in your field

- rapid publication on acceptance

- support for research data, including large and complex data types

- gold Open Access which fosters wider collaboration and increased citations

- maximum visibility for your research: over $100 \mathrm{M}$ website views per year

At $\mathrm{BMC}$, research is always in progress.

Learn more biomedcentral.com/submissions 